\title{
Impacto da suplementação com retinil palmitato no pós-parto imediato sobre os níveis de retinol do colostro ${ }^{1}$
}

\author{
Roberto Dimenstein ${ }^{2}$, Raquel Maria da Silva Lourenço $^{3}$ e Karla \\ Danielly da Silva Ribeiro ${ }^{4}$
}

Como citar Dimenstein R, Lourenço RMS, Ribeiro KDS. Impacto da suplementação com retinil palmitato no pósparto imediato sobre os níveis de retinol do colostro. Rev Panam Salud Publica. 2007;22(1):51-54.

RESUMO Objetivo. Avaliar o efeito da suplementação com retinil palmitato sobre os níveis de retinol no colostro, investigando a influência de variáveis maternas (idade, tipo de parto e estado nutricional bioquímico) sobre esses níveis.

Método. Participaram do estudo 33 nutrizes atendidas na Maternidade Escola Januário Cicco, Estado do Rio Grande do Norte, Brasil. Foram coletadas, nas primeiras horas após o parto, uma amostra de sangue e uma de colostro. Uma nova amostra de colostro foi coletada 6 horas após suplementação materna com 200000 UI de retinil palmitato. O retinol no sangue e colostro foi determinado por cromatografia líquida de alta eficiência.

Resultados. Os níveis médios de retinol no colostro antes da suplementação foram de 110,8 $\pm 82,3 \mu \mathrm{g} / \mathrm{dL}$, tendo atingido $164,4 \pm 106,5 \mu \mathrm{g} / \mathrm{dL}$ após a suplementação $(\mathrm{P}<0,025)$. Doze puérperas não responderam à suplementação ou tiveram um aumento inferior a $10 \%$ nos níveis de retinol no colostro; o nível de retinol sérico dessas mulheres foi significativamente mais baixo do que o daquelas que responderam à suplementação $(\mathrm{P}=0,024)$. Puérperas com parto cesáreo $(64 \%)$ tiveram níveis menores de retinol no soro $(\mathrm{P}=0,036)$, mas não no leite, em comparação a mulheres com parto normal. A idade não influenciou os níveis de retinol, nem no soro nem no leite.

Conclusões. A suplementação com retinil palmitato foi eficaz na elevação dos níveis de retinol no colostro, garantindo o fornecimento de quantidade suficiente de vitamina A para satisfazer o dobro das necessidades de retinol do recém-nascido.

Palavras-chave Colostro, período pós-parto, vitamina A, Brasil.

1 Artigo baseado na dissertação de mestrado de Raquel Maria da Silva Lourenço, "Influência da suplementação de retinol palmitato sobre os níveis de vitamina a do leite de puérperas saudáveis", apresentada em 2005 ao Programa de PósGraduação em Bioquímica da Universidade Federal do Rio Grande do Norte.

2 Universidade Federal do Rio Grande do Norte, Departamento de Bioquímica. Enviar correspondência a este autor no seguinte endereço: Departa- mento de Bioquímica, Centro de Biociências da Universidade Federal do Rio Grande do Norte, Avenida Senador Salgado Filho 3000, Bairro Lagoa Nova, CEP 59072-970, Natal, RN, Brasil. Telefone: +55-84-3215.3416/ramal 212; fax: +55-84-3211.9208; e-mail: robertod@ ufrnet.br

3 Nutricionista. E-mail: raquelms1@hotmail.com

4 Secretaria Municipal de Saúde de Natal, Natal (RN), Brasil. E-mail: karladaniellysr@yahoo.com.br
A vitamina A participa em processos vitais, como a diferenciação e a proliferação celular, o que a torna intimamente relacionada à remodelação óssea, à diferenciação epitelial e ao sistema imune. Sua deficiência se torna crítica durante períodos em que esses processos ocorrem de maneira intensa, como é 
o caso do crescimento e desenvolvimento fetal e na primeira infância (1).

Lactentes com menos de 6 meses de idade têm sido considerados como um grupo de grande vulnerabilidade à deficiência de vitamina A (2). Isso ocorre porque as reservas hepáticas da vitamina no lactente são muito limitadas ao nascimento, seja em decorrência de uma tendência à diminuição dos níveis de retinol sérico das gestantes, seja devido à existência de uma barreira seletiva placentária, que impede a passagem dessa vitamina para o feto (3). No intuito de elevar o consumo de vitamina A nos lactentes, a Organização Mundial da Saúde (OMS), o Fundo das Nações Unidas para a Infância (UNICEF) e o Grupo Consultivo Internacional sobre Vitamina A recomendam que, nas regiões onde a carência de vitamina A é endêmica, doses elevadas de vitamina A (200 000 UI) sejam administradas, em suplementação, às mulheres lactantes durante o período de 60 dias pós-parto (4). Todavia, os consultores da OMS afirmam que convém examinar os efeitos dessa suplementação na mãe e no lactente, de forma a avaliar as repercussões dessas estratégias sobre os programas, não esquecendo as vantagens de longo prazo, com a finalidade última de reduzir a mortalidade infantil.

No Brasil, nas últimas décadas, têm sido documentadas evidências de hipovitaminose A, sobretudo em estados da Região Nordeste; desde 1983, doses maciças de vitamina A vêm sendo distribuídas. Entretanto, só em 2002 esse programa foi ampliado para as puérperas residentes nos estados da Região Nordeste, Vale do Jequitinhonha (Minas Gerais) e três municípios do Estado de São Paulo, através da aplicação de uma megadose de vitamina A (200 $000 \mathrm{UI})$ por via oral no pós-parto imediato, momento da alta hospitalar (5).

Assim, o objetivo do presente trabalho foi avaliar o impacto da suplementação materna com retinol palmitato no pós-parto imediato sobre os níveis de retinol no colostro de puérperas saudáveis atendidas em uma maternidade pública de Natal, Estado do Rio Grande do Norte, Brasil.

\section{MATERIAIS E MÉTODOS}

O estudo foi realizado com aprovação do Comitê de Ética em Pesquisa da Universidade Federal do Rio Grande do Norte. Participaram da pesquisa 33 puérperas atendidas na Maternidade Escola Januário Cicco (MEJC), em Natal, que tiveram parto a termo e concepção única, sem histórico de doenças crônicas (hipertensão, diabetes ou outra). Informações como tipo de parto e idade materna foram obtidas dos respectivos prontuários.

As 33 parturientes foram selecionadas randomicamente até 12 horas após o parto. O tamanho da amostra, calculado no módulo Statcalc do programa Epi Info 3.32, foi estimado em no mínimo 25 sujeitos, para um nível de confiança de $95 \%$, considerando-se uma média de 250 partos por mês de mulheres que chegam para dar à luz na Maternidade Escola Januário Cicco.

No período matutino, foi coletada, inicialmente, uma amostra de $5 \mathrm{~mL}$ de sangue por punção venosa e, em seguida, uma amostra de $2 \mathrm{~mL}$ de leite, obtida por expressão manual de uma das mamas e com intervalo mínimo de 1 hora desde o último episódio de amamentação. Logo após a coleta do sangue e do leite, foi administrada às participantes uma megadose de 200000 UI $(60000 \mu \mathrm{g})$ de retinil palmitato. Uma segunda amostra de colostro de aproximadamente $2 \mathrm{~mL}$ foi obtida 6 horas após a suplementação. Esse intervalo foi definido com base no estudo de Azaïs-Braesco e Pascal (6), que indica que o processo de transporte até o fígado leva em torno de 5 horas. As amostras foram transportadas em tubos de polipropileno, devidamente protegidas da luz, e armazenadas a $-20{ }^{\circ} \mathrm{C}$ até a análise.

Para a extração do retinol do colostro e soro foi utilizado o método de Giuliano et al. (7). A fase hexânica obtida foi evaporada sob atmosfera de nitrogênio, em banho-maria, a $37^{\circ} \mathrm{C}$. Os extratos foram ressuspendidos em $1 \mathrm{~mL}$ de metanol (grau de pureza para cromatografia líquida de alta eficiência, CLAE). A concentração de retinol foi determinada por CLAE em um cromatógrafo LC-10 AD Shimadzu, acoplado a um detector SPD-10 A Shimadzu UV-VIS e integrador Chromatopac C-R6A Shimadzu com uma coluna LC Shim-pack CLC-ODS (M) 4,6 $\mathrm{mm} \times 25 \mathrm{~cm}$. A evolução do cromatograma ocorreu em fase móvel metanol $100 \%$ e fluxo de $1 \mathrm{~mL} / \mathrm{min}$. A identificação e a quantificação das amostras foram realizadas através da comparação com os tempos de retenção e com as áreas dos respectivos padrões. A concentração do padrão foi confirmada pelo coeficiente de extinção específico $(\varepsilon 1 \%, 1 \mathrm{~cm}=1850)$ em etanol absoluto e $\lambda=325 \mathrm{~nm}$ (8).

As análises estatísticas foram feitas utilizando-se o programa Statistica '99 Edition (StatSoft, Inc.), e os dados foram expressos como médias e desvio padrão. As diferenças entre as médias dos resultados normalmente distribuídos foram testadas através do teste $\mathrm{t}$ de Student, sendo significativas para $P<0,05$.

\section{RESULTADOS}

Os níveis médios de retinol encontrados no leite materno foram de 110,8 \pm $82,3 \mu \mathrm{g} / \mathrm{dL}$ antes da suplementação e $164,4 \pm 106,5 \mu \mathrm{g} / \mathrm{dL} 6$ horas após suplementação $(P<0,025$, teste $t$ de Student). Das 33 parturientes, 12 não responderam à suplementação ou tiveram um aumento inferior a $10 \%$ nos níveis de retinol no colostro em resposta à megadose. Quando comparamos os níveis de retinol no soro dessas 12 parturientes $(23,2 \pm 6,1 \mu \mathrm{g} / \mathrm{dL})$ com os níveis das mães que responderam à suplementação $(30,2 \pm 9,0 \mu \mathrm{g} / \mathrm{dL})$, encontramos uma diferença significativa $(P=0,024)$.

Também foi investigado o efeito da idade e do tipo de parto sobre a resposta à suplementação. A média de idade das mães foi de 26 anos e o tipo de parto mais freqüente foi o cesáreo (64\%). Não foi observada influência nem da idade, nem do tipo de parto, sobre os níveis de retinol no colostro ou no soro. Entretanto, foram observados níveis menores de retinol sérico no grupo de mulheres que fizeram cesárea $(25,1 \pm 7,6 \mu \mathrm{g} / \mathrm{dL})$ quando comparado ao grupo com parto normal $(32,4$ $\pm 11,4 \mu \mathrm{g} / \mathrm{dL})(P=0,036)$. 


\section{DISCUSSÃO}

A suplementação tem sido a medida de rotina utilizada na maternidade para tentar amenizar a carência de vitamina A nas mulheres, e evitar, dessa forma, os possíveis efeitos deletérios que essa carência pode causar às mães e a seus lactentes $(4,5)$. Considerando que os lactentes de até 6 meses de idade necessitam diariamente de 400 $\mu \mathrm{g}$ de retinol (9) e, em geral, consomem, nas primeiras semanas de vida, um volume médio diário de $500 \mathrm{~mL}$ de leite, o valor médio encontrado antes da suplementação, de 110,8 $\mu \mathrm{g} / \mathrm{dL}$, fornece $554 \mu \mathrm{g} /$ dia de vitamina A, seria, em princípio, suficiente para atender as necessidades do recémnascido (10). Por outro lado, o nível de retinol no colostro 6 horas após a suplementação era suficiente para fornecer o dobro da recomendação diária de retinol $(800 \mu \mathrm{g})$ aos recém-nascidos.

Os níveis de retinol no colostro das puérperas analisadas foram inferiores aos observados em mulheres na Índia $(131,6 \mu \mathrm{g} / \mathrm{dL})$ e Alemanha $(153 \mu \mathrm{g} / \mathrm{dL})$ $(11,12)$, e próximos aos valores encontrados em outro grupo na Índia (112 $\mu \mathrm{g} / \mathrm{dL})$, Cuba (102 $\mu \mathrm{g} / \mathrm{dL})$ e Brasil (93,1 $\mu \mathrm{g} / \mathrm{dL})$ (13-15). Entretanto, como esperado, o nível de retinol no colostro nas mulheres de Natal foi superior ao do leite maduro de lactantes do Sudeste brasileiro (40 $\mu \mathrm{g} / \mathrm{dL})(16)$.

Como no presente estudo, após suplementação com 200000 UI de vitamina A no pós-parto imediato, o retinol do colostro também foi elevado em mulheres de Bangladesh. Nas 24 horas após a suplementação, os níveis da vitamina eram, em média, 324,4 $\mu \mathrm{g}$ de retinol/dL, enquanto a do grupo controle era significativamente menor (84,37 $\mu \mathrm{g} / \mathrm{dL})$ (17). Em outro estudo, a concentração de retinol no colostro subiu de $110 \mu \mathrm{g} / \mathrm{dL}$ para 345,5 $\mu \mathrm{g} / \mathrm{dL}$
24 horas após a suplementação com 200000 UI de retinol no segundo dia pós-parto. Nesse momento, o grupo controle, que teve níveis basais semelhantes ao grupo teste, apresentou 84,7 $\mu \mathrm{g} / \mathrm{dL}$ como média de retinol no colostro (13).

Na presente amostra, algumas mães não demonstraram resposta 6 horas após a suplementação, enquanto outras ultrapassaram $200 \%$ de aumento. $\mathrm{Na}$ tentativa de elucidar a forma como a suplementação atuou, deve-se considerar o fato de que a dose recomendada é baseada nas necessidades de lactantes que possuem um bom estado nutricional inicial, boa saúde, consumo dietético adequado para atingir as necessidades basais de vitamina A e ingestão adicional de retinol relativa à fase de lactação $(2,11,18)$. Com isso, é possível considerar que parte das mães não possuía algum dos requisitos fundamentais para obtenção do êxito esperado com a suplementação. Não se deve desprezar, entretanto, a existência de outros fatores intimamente associados à absorção, estocagem, transporte e utilização do retinol: a quantidade disponível de proteína transportadora de retinol, que depende de proteínas da dieta e da disponibilidade de zinco, a presença de parasitas, problemas do trato gastrintestinal, o consumo dietético, todos são fatores que podem ter influenciado o aproveitamento da dose suplementar $(4,13,19,20)$.

Observamos uma diferença significativa entre os níveis de retinol no soro das mulheres que responderam à suplementação quando comparadas às que não responderam. Esses resultados mostram a influência dos níveis de retinol sérico sobre a resposta à suplementação e podem contribuir como importante indicador bioquímico, auxiliando na decisão da utilização de uma segunda megadose de vitamina
A, conforme sugestão da Organização Pan-Americana da Saúde (OPAS) (21).

Quando analisado o efeito da idade e do tipo de parto sobre a resposta à suplementação, não foi observado um impacto sobre o nível de retinol no colostro. Porém, independentemente do efeito da suplementação, foram observadas diferenças significativas entre os níveis de retinol sérico nas mulheres que fizeram cesárea ou parto normal. Esse resultado pode estar associado ao tipo de medicamento utilizado no momento do parto ou ao estresse provocado pelo ato cirúrgico, que provoca um aumento das proteínas de fase aguda, podendo ocasionar uma diminuição nos níveis séricos de proteína transportadora de retinol e, conseqüentemente, nos níveis de retinol (22).

O período do pós-parto imediato representa um momento tanto programático quanto biológico de oportunidade para beneficiar a mulher em idade reprodutiva com altas doses de vitamina $\mathrm{A}$, tendo em vista que uma grande proporção de mulheres está em contato com o sistema de saúde $(13,23)$. Além disso, a megadose parece beneficiar também a criança $(10$, 24). O presente trabalho demonstrou que a suplementação de vitamina A foi capaz de elevar os níveis de retinol no colostro, fornecendo quantidade suficiente para satisfazer o dobro das necessidades de retinol do recémnascido. Além disso, conclui-se que os níveis basais de retinol sérico influenciam a resposta à suplementação.

Agradecimentos. Os autores agradecem à Universidade Federal do Rio Grande do Norte e à CAPES pelo apoio financeiro e pelo incentivo ao desenvolvimento da pesquisa; e à Maternidade Escola Januário Cicco pela permissão para a realização do presente estudo.

\section{REFERÊNCIAS}

1. Blomhoff R. Vitamin A and carotenoid toxicity. Food Nutr Bull. 2001;22(3):320-34.

2. Rice AL, Stoltzfus RJ, de Francisco A, Chakraborty J, Kjolhede CL, Wahed MA. Maternal Vitamin A or beta-carotene supplementation in lactating Bangladeshi women bene- fits mothers and infants but does not prevent subclinical deficiency. J Nutr. 1999;129(2): 356-65.

3. Rondó PHC, Abbott R, Rodrigues LC, Tomkins AM. Vitamin A, folate, and iron concentrations in cord and maternal blood of intra- uterine growth retarded and appropriate birth weight babies. Eur J Clin Nutr. 1995; 49(6):391-9.

4. World Health Organization (WHO). Vitamina A na gestação e lactação: recomendações e relatório de uma consultoria. Brasília: Centro 
Colaborador de Alimentação e Nutrição do Nordeste I; 2001. (Série micronutrientes).

5. Brasil, Ministério da Saúde. Projeto Suplementação de Megadose de Vitamina " $\mathrm{A}$ " no pós-parto imediato nas maternidades/ hospitais. Brasília: Ministério da Saúde; 2002. Disponível em: http://dtr2004.saude.gov.br/ nutricao/mn/vita/docs/projeto_vita.pdf. Acessado em abril de 2006

6. Azaïs-Braesco V, Pascal G. Vitamin A in pregnancy: requirements and safety limits. Am J Clin Nutr. 2000;71(5 Suppl):1325S-33S.

7. Giuliano AR, Neilson EM, Kelly BE, Canfield LM. Simultaneous quantitation and separation of carotenoids and retinol in human milk by high-performance liquid chromatography. Methods Enzymol. 1992;213:391-9.

8. Nierenberg DW, Nann SL. A method for determining concentrations of retinol, tocopherol, and five carotenoids in human plasma and tissue samples. Am J Clin Nutr. 1992; 56(2):417-26.

9. Institute of Medicine. Dietary reference intakes for vitamin A, vitamin $\mathrm{K}$, arsenic, boron, chromium, copper, iodine, iron, manganese, molybdenum, nickel, silicon, vanadium, and zinc. Washington, DC: National Academy Press; 2001.

10. Ross JS, Harvey PWJ. Contribution of breastfeeding to vitamin A nutrition of infants: a simulation model. Bull World Health Organ. 2003;81(2):80-6

11. Bhaskaram P, Balakrishna N, Nair KM, Sivakumar B. Vitamin A deficiency in infants: effects of postnatal maternal vitamin A supple- mentation on the growth and vitamin A status. Nutr Res. 2000;20(6):769-78

12. Schweigert FJ, Bathe K, Chen F, Büscher U, Dudenhausen JW. Effect of the stage of lactation in humans on carotenoid levels in milk, blood plasma and plasma lipoprotein fractions. Eur J Nutr. 2004;43(1):39-44.

13. Basu S, Sengupta B, Paladhi PK. Single megadose vitamin A supplementation of Indian mothers and morbidity in breastfed young infants. Postgrad Med J. 2003;79(933):397-402.

14. Macias C, Schweigert FJ. Changes in the concentration of carotenoids, vitamin A, alphatocopherol and total lipids in human milk throughout early lactation. Ann Nutr Metab. 2001;45(2):82-5.

15. Dimenstein R, Simplício JL, Ribeiro KDS, Melo ILP. Influência de variáveis socioeconômicas e de saúde materno-infantil sobre os níveis de retinol no colostro humano. J Pediatr (Rio J). 2003;79(6):513-8.

16. Meneses F, Torres AG, Trugo NM. Influence of recent dietary intake on plasma and breast milk levels of carotenoids and retinol in Brazilian nursing women. Adv Exp Med Biol. 2004;554:351-4.

17. Roy SK, Islam A, Molla A, Akramuzzaman SM, Jahan F, Fuchs G. Impact of a single megadose of vitamin A at delivery on breastmilk of mothers and morbidity of their infants. Eur J Clin Nutr. 1997;51(5):302-7.

18. Canfield LM, Taren DL, Kaminsky RG, Mahal Z. Short-term beta-carotene supplementation of lactating mothers consuming diets low in vitamin A. J Nutr Biochem. 1999;10(9):532-8.
19. Ramalho RA, Saunders C, Paiva F, Accioly E, Cardoso LO, Natalizi D. Estado de vitamina A de puérperas e recém-nascidos e estado antropométrico materno. Rev Cienc Med PUCCAMP. 2001;10(1):5-10.

20. Stoltzfus RJ, Hakimi M, Miller KW, Rasmussen KM, Dawiesah S, Habicht JP, et al. High dose vitamin A supplementation of breastfeeding Indonesian mothers: effects on the vitamin A status of mother and infant. J Nutr 1993;123(4):666-75.

21. Organización Panamericana de la Salud. La provisión de la vitamina A complementa la inmunización y otros contactos de salud para los niños 6-59 meses y las mujeres hasta 6 semanas de posparto: una guía para los trabajadores de salud. 2a ed. Washington: OPAS; 2001.

22. de Pee S, Dary O. Biochemical indicators of vitamin A deficiency: serum retinol and serum retinol binding protein. J Nutr. 2002;132(9 Suppl):2895S-2901S.

23. Humphrey JH, Ichord RN. Safety of vitamin A supplementation of postpartum women and young children. Food Nutr Bull. 2001; 22(3):311-9.

24. World Health Organization, United Nations Children's Fund, International Vitamin A Consultative Group. Vitamin A supplements: a guide to their use in the treatment and prevention of vitamin A deficiency and xerophthalmia. 2a ed. Genebra: WHO; 1997.

Manuscrito recebido em 29 de outubro 2006. Aceito em versão revisada em 21 de maio de 2007.
ABSTRACT

Impact on colostrum retinol levels of immediate postpartum supplementation with retinyl palmitate
Objective. To assess the effect of retinyl palmitate supplementation on colostrum retinol levels, investigating the influence of maternal variables (age, type of delivery, and biochemical nutritional status) on these levels.

Method. The study included 33 mothers receiving care at the Januário Cicco Maternity School, in the city of Natal, Rio Grande do Norte, Brazil. In the first hours after delivery, blood and colostrum samples were collected. Another colostrum sample was collected six hours after maternal supplementation with 200000 IU of retinyl palmitate. Serum and colostrum retinol levels were determined by high-performance liquid chromatography.

Results. The mean retinol level in colostrum before the supplementation was 110.8 $\pm 82.3 \mu \mathrm{g} / \mathrm{dL}$, and after supplementation it was $164.4 \pm 106.5 \mu \mathrm{g} / \mathrm{dL}(P<0.025)$. Of the 33 mothers, 12 of them either did not respond to supplementation or had an increase of less than $10 \%$ in colostrum retinol levels; serum retinol in these women was significantly lower as compared to the responders $(P=0.024)$. In comparison to women with a normal delivery, the mothers who underwent cesarean delivery $(64 \%)$ had lower serum retinol levels, but not lower colostrum retinol levels $(P=0.036)$. Maternal age did not influence retinol levels in either serum or colostrum.

Conclusion. The increase in colostrum retinol levels following vitamin A supplementation was sufficient to guarantee double the retinol requirements of a newborn infant.

Key words Vitamin A, dietary supplements, colostrum, postpartum period, Brazil. 